\title{
Classification retrieval method on planter design
}

\author{
Jing Zang ${ }^{1, a}$, Lidong $\mathrm{Fu}^{1}$ and Chenghua $\mathrm{Li}^{2}$ \\ ${ }^{1}$ Shenyang Ligong University, School of Information Science and Engineering, 110159, Shenyang, China \\ ${ }^{2}$ Shenyang Ligong University, School of Mechanical Engineering, 110159, Shenyang, China
}

\begin{abstract}
In order to increase efficiency in digital design on single and small batch Spade Punch Planter, The structure and attribute set of all-level case was described based on case base and a improved the classification retrieval strategy on the multi-level case for finding optimal reused case were developed. A membership function algorithm was used to determine the scheme category of the new design problem, and a SM model based on extension matterelement was used for computing similarity between history case and design problem case after the multi-level matching techniques on new problem attributes inside the scheme category. Experiment with the matter-element case of soil-opener verified the accuracy, stability and good resolution in case of retrieval algorithm, which illustrates the research provide the method of the classification retrieval strategy was efficient and feasible.
\end{abstract}

Keywords: planter; CBR; similarity; multi-hierarchical cases.

\section{Introduction}

Case-based design [1,2] is a well-known artificial intelligence technique that emphasizes finding appropriate past experience to the solution of new problems, Cases with the highest similarity value to the new case would be adopted. Modification may take place to enhance the case suitability. There are four activities[3,4] in the CBD module:(1)Retrieve similar cases to the problem description.(2) Reuse a solution suggested by a similar case.(3) Revise or adapt that solution to better fit the new problem if necessary.(4) Retain the new solution once it has been confirmed or validated.

Case-based design in this paper comprises retrieving the most similar previous cases from the case base to provide solution of the new planter design, which solve the heavy tasks and lack of time in the planter's digital design in single-part or small-lot production to a large extent.

Many researchers have proposed various retrieval techniques have been developed, such as the famous mix neural net-works [5], gray-relational algorithms 6] or extenics method [7, 8].

Many classic retrieval strategies $[9,10]$ is difficult to do case with sophisticated attribute in case retrieval problems because of its multi-level design process and many kinds of attributes, the multilevel classification retrieval strategy is proposed

\footnotetext{
a Corresponding author: zang_jing@163.com
} 


\section{Representation method of the multi-hierarchical case}

If the case is represented all attributes of the planter, each case have too many features is tedious, and It is incredible to use all of them to represent a case for retrieving due to the reason that many redundant features may cost a large number of computational times and deteriorate system performance.

Based on the axiomatic design, historical design case is decomposed by the extension modular approach in weak-coupling and multi-level way. And at the same time the structure of each sub-case and multi-level case base is decided, ultimately determine all the design attributes of the planter structure.

Each Multi-hierarchical case in the case memory contains three parts: $C=(D, C c, C s), D$ is the parent-case number, which is allocated by the system in order to provide the attribute of its parent case for each case; Cc is problem description, which is used to describe the situations when the case happened; Cs is solution description, which is used to represent the results of the corresponding cases. If $\mathrm{C}$ is the first-level case, there is no the parent-case number, $\mathrm{D}$ is null. $\widetilde{C}=\left\{\widetilde{C}_{1}, \widetilde{C}_{2}, \cdots, \widetilde{C}_{s}\right\}$ is denoted the set of the sub-case. $\widetilde{C}_{c}=\left(D, C c_{1}, C c_{2}, \cdots, C c_{n}\right)$ denotes the attribute set of historical sub-case $\tilde{C}_{i}$.

\section{The vector of attribute with regard to the Multi-hierarchical case}

The vector of attribute divide into two types: The vector of attribute of the root case and the vector of attribute of the sub-case.

\subsection{The attributes vector of Scheme design case}

The attributes of scheme-level case consist of the key attributes of design sub-case, which are decided the classification for the sub-case, is the multi-level design case of the root-level case.

Scheme design case is divided into $\mathrm{K}$ classes, the attributes of Scheme-level case consist of the key attributes of its k-classes sub-cases, $\tilde{C}_{c}(1)=\left(C c_{1}(1), C c_{2}(1), \cdots, C c_{n}(1)\right)$ denotes the attribute vector of Scheme design case, deleting repeating sequences. $\mathrm{P}(1)=\left(\mathrm{p}_{1}, \mathrm{p}_{2}, \ldots, \mathrm{p}_{\mathrm{M}}\right)$ denotes a vector of attribute with regard to the problem situation of Scheme case; $\mathrm{VP}(1)=\left(\mathrm{V}_{\mathrm{P} 1}, \mathrm{~V}_{\mathrm{P} 2},,, \mathrm{~V}_{\mathrm{PM}}\right)$ denotes a vector of attribute values with regard to its problem situation.

\subsection{The attribute vector of the sub-case}

Scheme design case is decomposed into multi-level cases, $\widetilde{C}_{c}(m)=\left(D(m), C c_{1}(m), C c_{2}(m), \cdots, C c_{n}(m)\right)$ denotes the attribute vector of the mth-level sub-case, $P(m)=\left(p_{1}, p_{2}, \ldots, p_{s}\right)$ denotes a vector of attribute with regard to the problem situation of the mth-level sub-case.

\subsection{The vector of attribute with regard to the problem situation of object case}

$\mathrm{P}_{0}$ denotes the requirements of the new planter design, we turn the requirement analysis into the problem attributes of object cases, which consist of the functional features, performance characteristics and design constraints. $\mathrm{P}_{0}(\mathrm{~m})=\left(\mathrm{p}_{1}, \mathrm{p}_{2}, \ldots, \mathrm{p}_{\mathrm{s}}\right)$ denotes a vector of attribute with regard to the problem situation of object case. 


\section{Retrieval model}

Step 1 According to the multi-level case in case base of the planter, the attribute vector of each subcase in different level is determined, included: functional features, performance characteristics, design constraints, technical parameters and structure parameters for the design solution, which is discussed in section 2;

Step 2 According to technical requirements and design requirements of the target design, we turn the key atrributes with regard to the design problem into the feature set and the corresponding vector, in accordance with section 3.1;

Step3 To merge the same attributes in case at all levels and get the key attribute set of the schemeclass case, its vector and determine the key attribute set of the sub-cases, their vector ;

Step4 To calculate membership degree with the vector of attribute values with regard to the problem situation of the scheme-case and a vector of attribute values with regard to the problem situation of target case, and determine the class of the design problems;

Step5 To match the attributes of the target case with the sub-case and determine the set of the attributes of the mth-level sub-case;

Step6 To compute the grey copositive SM with regard to multi-level cases based on Step4, Step5, and get the best similar cases.

\section{Retrieval algorithm}

\subsection{The category on the new design problem}

Based on design requirements, to determine the scheme category of the new design problem, using membership function $F_{k}$ to measure.

$F_{k}$ denotes the membership function of the problem attribute vector of the target case $P_{0}(1)$ with regard to the vector of the problem situation of the scheme-case.

$\mathrm{F}_{\mathrm{k}}=$ the number of common attributes with regard to the problem attribute of the target case and the scheme-case/ the number of the problem attributes of the target case

$F_{k}=1$ means the design problem and the scheme-level case match exactly; $F_{k}=0$ means the design problem and the scheme-level case do not match exactly;

\subsection{The multi-level matching techniques on new problem attributes}

According to the membership $F_{k}$ of each sub-class case of the scheme-level case, get the scheme classification of design problem and determine the scheme-level case and the mth-level sub-case.

The multi-level matching process on new problem attributes:

Step 1 matching each problem attribute of the target case to the problem attributes of the $(m+1)$ th case;

Step 2 If the mth level sub-case is found, the indexed-code of the $(m+1)$ th case is set to 1 ;

Step3 To match the $(\mathrm{m}+1)$ th sub-case to the problem of the target case, the indexed-code of the $(\mathrm{m}+2)$ th case is set to 1 ;

Step 4 To match the next level sub-case to the problem of the target case and so on, determine the attributes of each sub-case which satisfied the problem of the target case

\subsection{The similarity between the target matter-element case and the multi-level historical case}

The SM between the matter-element of problem and the history case is given by formula (1), 


$$
\operatorname{sim}_{j}\left(Z_{0}, Z_{i}^{r}\right)=\frac{\min _{r} \min _{i}\left|p_{i j}-p_{0 j}\right|+\beta \max _{r} \max _{i}\left|p_{i j}-p_{0 j}\right|}{\left|p_{i j}-p_{0 j}\right|+\beta \max _{r} \max _{i}\left|p_{i j}-p_{o j}\right|},
$$

$\beta$ is the distinguishing coefficient, $\beta=0.5$

The weight is determined by AHP, $w^{2}=(0.198,0.182,0.211,0.069,0.085,0.089,0.102,0.098,0.089)$, the compositive approach of similarity computation between the multi-level case $\mathrm{Z}_{\mathrm{i}}^{\mathrm{r}}$ and the target case $\mathrm{Z}_{0}$.

$$
\operatorname{sim}\left(Z_{0}, Z_{i}^{r}\right)=\sum_{j=i}^{m} w_{j} * \operatorname{sim}_{j}\left(Z_{0}, Z_{i}^{r}\right)
$$

\section{Application example}

The retrieval method is used to the soil-opener and its sub-case design case base of Spade Punch Planter. There are 3 sub-cases spade-wheel, rotational axis, bolt connection of 2nd-cases of soilopener , and 9 attributes, is shown by table 1 and the matter-element-values of problem and the history case soil-opener and its sub-case is in table1,table2. The data was stored in multi-database created by SQL Server 2008, and the algorithm was realized in c\#.

Step1 Based on design requirements of the new planter, to determine the scheme category of the new design problem,

Step2 Convert the requirements of user to the problem attribute sets according to the opinions from the experts, and match the attribute set of problem case attribute set at all levels, and the corresponding attribute-values .

Step3 Based on Step2, set up the matter-element -attribute-vector of problem and the history case, and compute the SM between them by formula (1),the result is shown by table 3;

\begin{tabular}{|c|c|c|c|c|}
\hline $\begin{array}{l}\text { Weights } \\
\text { of case }\end{array}$ & $\begin{array}{l}\text { 3rd-level } \\
\text { sub-case }\end{array}$ & Attributes of case & $\begin{array}{l}\text { Signal of } \\
\text { Attributes }\end{array}$ & $\begin{array}{l}\text { Weights Of } \\
\text { Attributes }\end{array}$ \\
\hline \multirow{5}{*}{0.5} & \multirow{5}{*}{$\begin{array}{l}\text { spade- } \\
\text { wheel }\end{array}$} & Plate diamet(mm) & A1 & 0.3 \\
\hline & & bore number & $\mathrm{A} 2$ & 0.3 \\
\hline & & Spade thicken(mm) & A3 & 0.1 \\
\hline & & spade height(mm) & A4 & 0.2 \\
\hline & & Spade width(mm) & A5 & 0.1 \\
\hline \multirow{2}{*}{0.4} & \multirow{2}{*}{$\begin{array}{l}\text { rotational } \\
\text { axis }\end{array}$} & Outside diameter(mm) & A6 & 0.5 \\
\hline & & Inside diameter(mm) & A7 & 0.5 \\
\hline \multirow{2}{*}{0.1} & \multirow{2}{*}{$\begin{array}{c}\text { bolt } \\
\text { connection }\end{array}$} & Major diameter(mm) & A8 & 0.5 \\
\hline & & Length(mm) & A9 & 0.5 \\
\hline
\end{tabular}

Table 1. The matter-element-attributes of problem and the history case soil-opener and its sub-case 
Table 2. The matter-element-values of problem and the history case soil-opener and its sub-case

\begin{tabular}{|c|c|c|c|c|c|}
\hline \multirow{2}{*}{$\begin{array}{c}\text { Signal } \\
\text { Of } \\
\text { Attributes }\end{array}$} & \multicolumn{4}{|c|}{$\begin{array}{c}\text { 2nd-cases of matter-element of } \\
\text { soil-opener }\end{array}$} & \multirow{2}{*}{$\begin{array}{c}\text { Target } \\
\text { case }\end{array}$} \\
\cline { 2 - 5 } & $\mathrm{c} 1$ & $\mathrm{c} 2$ & $\mathrm{c} 3$ & $\mathrm{c} 4$ & \\
\hline A1 & 650 & 550 & 670 & 450 & {$[630,640]$} \\
\hline A2 & 15 & 10 & 12 & 10 & {$[14,15]$} \\
\hline A3 & 118 & 78 & 96 & 68 & {$[115,120]$} \\
\hline A4 & 61 & 33 & 38 & 30 & {$[56,60]$} \\
\hline A5 & 88 & 96 & 108 & 66 & {$[86,60]$} \\
\hline A6 & 80 & 90 & 70 & 70 & 90 \\
\hline A7 & 60 & 70 & 50 & 50 & 70 \\
\hline A8 & 10 & 10 & 16 & 12 & 12 \\
\hline A9 & 40 & 40 & 60 & 50 & 50 \\
\hline
\end{tabular}

Table 3 .The attribute similarity sequence in case of soil-opener and its sub-case

\begin{tabular}{|c|c|c|c|}
\hline SM1 & SM2 & SM3 & SM 4 \\
\hline 0.948 & 0.752 & 0.836 & 0.752 \\
\hline 1.000 & 0.467 & 0.854 & 0.467 \\
\hline 0.965 & 0.701 & 0.762 & 0.701 \\
\hline 0.992 & 0.596 & 0.678 & 0.596 \\
\hline 1.000 & 0.902 & 0.842 & 0.902 \\
\hline 0.891 & 1.000 & 0.763 & 0.763 \\
\hline 0.866 & 1.000 & 0.682 & 0.682 \\
\hline 0.966 & 0.966 & 0.750 & 1.000 \\
\hline 0.800 & 1.000 & 0.874 & 1.000 \\
\hline
\end{tabular}

Step4 the SM between the matter-element of problem case and the history case is given by formula (2), the result is described as follows:

$$
\operatorname{Sim}\left(Z_{0}, Z_{i}^{r}\right)=(0.939,0.752,0.782,0,574)
$$

The result with the matter-element case of soil-opener show that CASE1 may be useful in case adaption, which need the next the judgement.

The calculation result shows that the accuracy of the similarity matrix of is higher than the literature [10], and the similarity between the selected case 1, 3 and the target case of is relative taller; making analysis on the CASE1 attributes, which is closer to the target case, so we can revise the adjustable parameters of CASE1, accordingly to some adaption strategy and the plant design requirements to complete new plant's design.

\section{Conclusions}

The case similarity calculation model based on extension matter-element case improves the similarity evaluation on spade punch planter with mixed attributes and different formats of attribute values, to ensure retrieval accuracy in cases of different design problems. 
In this method, the attribute similarity of multi-level cases is calculated, which is different from the previous work [10], the propose method has more clear logic computation procedure, and developed a new idea for case adaption.

The new retrieval model is efficient and feasible.

\section{Acknowledgements}

In this paper, the research is sponsored by the National Science Foundation of China (Project No. 51075282), the Public Research Funds of Liaoning (Project No.2014002006) and Key Disciplines and Key Laboratory Open Fund for Shenyang Ligong University (Project No.4771004kfx14).

\section{References}

1. Fan Zh.P., Li Y.H., Wang X..h..Expert Systems with Applications,41,9(2014)

2. Qi, J., Hu, J., Peng, Y. H., Wang, W., \& Zhang, Z.. Expert Systems with Applications, 36,10(2009)

3. S.I. Laoa, K.L. Choya, G.T.S. Hoa, Richard C.M. Yamb,1, Y.C. Tsima, T.C. Poon, Expert Systems with Applications, 3911 (2012)

4. S.Z. Dogan, D. Arditi, H.M. Gnaydin, Journal of Construction Engineering and Management 134,2 146-152(2008).

5. K.H. Im, S.C. Park. Expert Systems with Applications 32,9 (2007)

6. Hao B., Song Y., Hu Y.l..Liu H.j..Modular Machine Tool \& Automatic Manufacturing Technique. 9,4,(2014)

7. Jia Yanhua, MO Rong, YANG Haicheng, WANG Weizhen. Computer Engineering and Applications, 49,2,258-260 (2013)

8. Chen J.J., Ge R.H., Liu D.f., Wang B.,Manufacturing automation, 34,4,85-88 (2012)

9. Chen J., Gong Zh.W., Liu L., Cheng Y.. MACHINE TOOL \& HYDRAULICS,40 6(2012)

10. Jing Z., Qian-qian G.,Advanced Technologies in Manufacturing, engineering and materials,part1, 180-184(2013) 